\title{
Zum Tod von Rolf Schwendter
}

Rolf Schwendter ist am 21. Juli gestorben. Er wurde 73 Jahre alt. Die Themen des in Wien geborenen Universalgenies reichten von „abweichendem Verhalten“ bis zur Esskultur - beides erprobte er ausgiebig in der Praxis, und seine Manuskripte waren so ungewöhnlich wie sein Auftreten. Seit 1975 war Schwendter Professor für Devianzforschung an der Universität Kassel. Neben seinem Grundlagenwerk „Theorie der Subkultur“ veröffentlichte er zahlreiche Schriften zu Themen wie „Geschichte der Zukunft“, „Einführung in die Soziale Therapie“, „Sittengeschichte des Alltags“ ebenso wie Gedichtbände und Kochbücher. In den 1980er und 90er Jahren war er auch Sozial-Extra-Autor.

Die Soziale Arbeit hat er vor allem durch die Gründung der AG Spak und zahlreicher weiterer Initiativen und Netzwerke zur Entwicklung alternativer Formen der Hilfe und Intervention weit über den Bereich der Sozialtherapie hinaus beeinflusst. Die Kollegen vom Promedia-Verlag haben seine Persönlichkeit und sein Werk mit folgenden Worten gewürdigt: „Mit seinem Tod verliert die linke und alternative Bewegung und mit ihr die gesamte deutschsprachige Öffentlichkeit einen der vielseitigsten Kritiker menschlicher Entfremdungen im postindustriellen Zeitalter. Schwendter war Sänger, Trommler und Wortspieler; er war Philosoph, Jurist und Historiker; Autor, Lehrer und Forscher; er sprach ungarisch, wienerisch und deutsch. Seine kontemplative Kochkunst war legendär."

Er selbst hat seinen Werdegang ebenso bescheiden wie selbstbewusst beschrieben: „Aus dem, was in der dialektischen Lehre ,abstrakte Negation' heißt, bin ich dazu gekommen, mich für alles zu interessieren, was abwich. Was ich in meiner Jugend nicht wissen konnte, ist, dass das irgendwann einmal mein Beruf werden sollte... Meine gesamte bildungsgemäße Sozialisation lief darauf hinaus, nicht wegen, sondern trotz der Bildungsinstitutionen zu lernen und dieses dann im formalen Bildungsapparat irgendwie legitimieren zu lassen... Ich hoffe, mit meiner Aussage darüber nicht zu einem Mythos beizutragen. Aber ich habe in der Tat als 18jähriger Schüler, noch vor der Matura, einen Plan aufgestellt, dass ich bis zu meinem 28. Lebensjahr die drei Studien jur., rer. pol. und phil. absolviert haben wollte. “1

Und das hat er auch tatsächlich getan. Die Frage danach, wie er im Rückblick seine Erfolge und die Rezeption seiner umfangreichen Publikationstätigkeit einschätzt, bezeugt den eklatanten Mangel an Eitelkeit, der Rolf Schwendter immer ausgezeichnet hat: „Ich muss an mich denselben Anspruch an Selbstreflexivität stellen, den ich an andere stelle. Es ist klar, dass Leute wie ich sich immer zu wenig rezipiert vorkommen. Mal denke ich, dass das Interesse an meinen Themen stärker ist als die öffentliche Meinung suggeriert. Vor allem, wenn ich über Zukunftsforschung gesprochen habe, war der Saal meistens berstend voll. Ein anderes Mal war es dann nicht so. Ganz sicher hat aber das Medium der Zukunftswerkstätten zwischenzeitlich eine Breitenwirkung her-

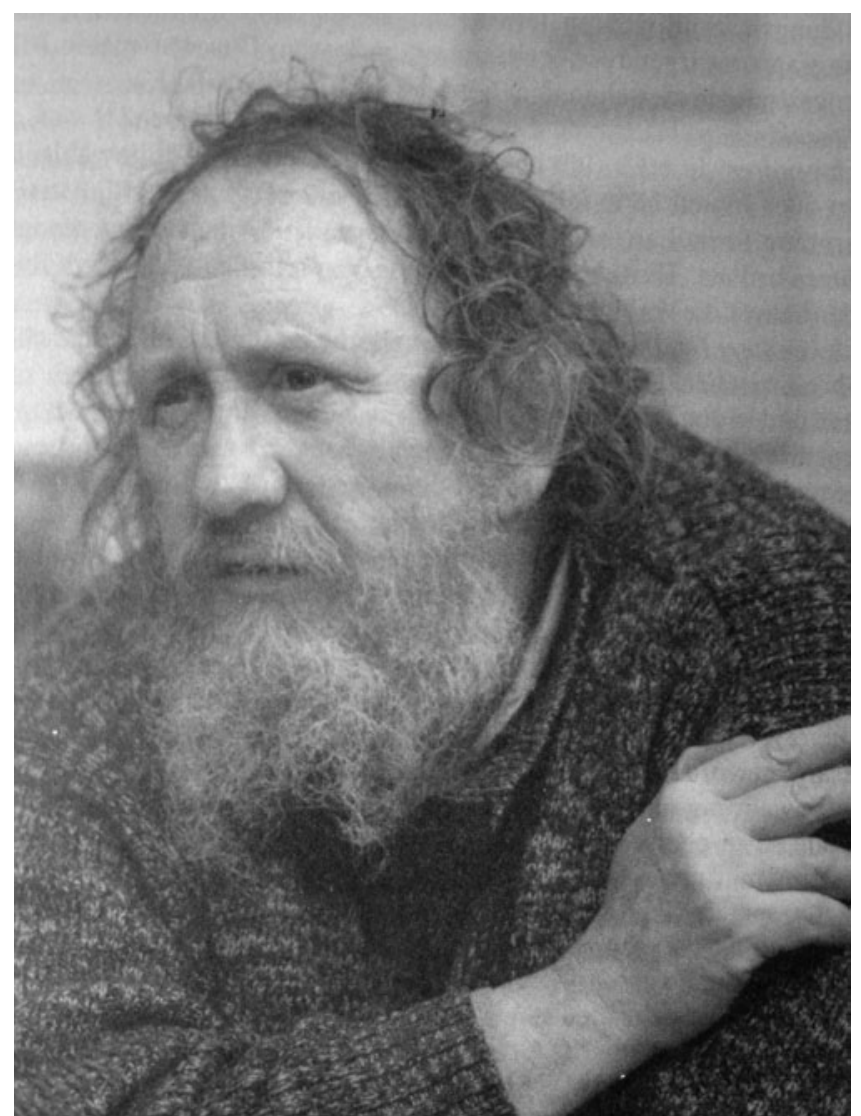

Rolf Schwendter

Foto: Günther Borgmann

gestellt, die über die Grenzen der alternativen Bildung weit hinausgegangen ist.“

Rolf Schwendter, der immer über alle Grenzen hinausgegangen ist, um zu mehr Menschlichkeit, Kultur, Demokratie und wissenschaftlicher Erkenntnis beizutragen, wird uns sehr fehlen.

Sabine Hering/Bernhard Schön

1. Die Statements von Rolf Schwendter sowie das Foto von ihm entstammen dem Buch: Sabine Hering/Hans-Georg Lützenkirchen (1996), Wohin führt der lange Marsch? Die politische Erwachsenenbildung der 68er. Gespräche. Frankfurt/Main. 